\title{
A Super-Absorbent Polymer Combination Promotes Bacterial Aggressiveness Uncoupled from the Epiphytic Population
}

\author{
Boyoung Lee ${ }^{1}$, Dalsoo $\mathrm{Kim}^{2}$ and Choong-Min Ryu ${ }^{1,3 *}$ \\ ${ }^{I}$ Systems Microbiology Research Center, KRIBB, Daejeon 305-333, Korea \\ ${ }^{2}$ Panagene, Daejeon 305-510, Korea \\ ${ }^{3}$ Field of Functional Genomics, University of Science and Technology, Daejeon 305-333, Korea \\ (Received on July 29, 2008; Accepted on August 14, 2008)
}

Plant leaf surface is an important niche for diverse epiphytic microbes, including bacteria and fungi. Plant leaf surface plays a critical frontline defense against pathogen infections. The objective of our study was to evaluate the effectiveness of a starch-based super-absorbent polymer (SAP) combination, which enhances water potential and nutrient availability to plant leaves. We evaluated the effect of SAP on the maintenance of bacterial populations. In order to monitor bacterial populations in situ, a SAP mixture containing Pseudomonas syringae pv. tabaci that expressed recombinant green fluorescent protein (GFPuv) was spray-challenged onto whole leaves of Nicotiana benthamiana. The SAP combination treatment enhanced bacterial robustness, as indicated by disease severity and incidence. Unexpectedly, bacterial numbers were not significantly different between leaves treated with the SAP combination and those treated with water alone. Furthermore, young leaves treated with the SAP combination had more severe symptoms and a greater number of bacterial spots caused by primary and secondary infections compared to young leaves treated with the water control. In contrast, bacterial cell numbers did not statistically differ between the two groups, which indicated that measurement of viable GFP-based bacterial spots may provide a more sensitive methodology for assessing virulence of bacterial pathogens than methods that require dilution plating following maceration of bacterialinoculated leaf tissue. Our study suggests that the SAP combination successfully increased bacterial aggressiveness, which could either be used to promote the ability of biological agents to control weedy plants or increase the robustness of saprophytic epiphytes against competition from potentially harmful microbes.

Keywords : aggressiveness, epiphyte, Nicotiana benthamiana, Pseudomonas syringae

Plant leaf surface provides an important habitat for many microbes. Numerous microbes, including pathogens and

\footnotetext{
*Corresponding author.

Phone) +82-42-879-8229, FAX) +82-42-860-4488

E-mail) cmryu@kribb.re.kr
}

saprophytes, can survive and even multiply to various degrees even though the phyllosphere environment is not favorable to such epiphytes, due to ultraviolet irradiation and limited supplies of water and nutrients (Beattie and Lindow, 1995; Dulla and Lindow, 2008; Gunasekera and Paul, 2007). Among the microbes on plant leaves, plant pathogenic bacteria have been intensively studied because they are casual factors for the significant yield losses of certain plant (host) species (Hirano and Upper, 2000; Krzymowska et al., 2007). In addition, bacterial contamination on the surface of fruit and vegetables by Salmonella typhimurium, S. enterica, and Escherichia coli $\mathrm{O} 157$ has recently increased public interest because this contamination poses an important human health issue (Barak and Liang, 2008; Goodin et al., 2008; Heaton and Jones, 2007; Whipps et al., 2008). Besides plant and human pathogenic bacteria, several groups of saprophytic epiphytic bacteria can thrive throughout their life cycle with as many as 1 to 10 million cells per leaf surface without causing any symptoms and without colonizing the insides of plant leaf tissues, such as within the apoplast (Kinkel, 1997).

In order to successfully establish a bacterial population on the plant leaf surface, epiphytic bacteria require three functional processes: 1) immigration (landing of viable bacterial cells on the leaf), 2) growth (an increased number of bacteria via proliferation under favorable conditions), and 3) death (bacterial cell death under harsh conditions) (Kinkel, 1997). In the present study, Pseudomonas syringae was utilized as a model bacterium for understanding such bacterial population dynamics because this bacterial species grows abundantly on diverse plant surfaces, is a wellcharacterized leaf pathogen or a saprophyte, depending on the host plant, and is a more aggressive colonizer than gram-positive bacteria such as Bacillus spp. (Hirano and Upper, 2000; Maduel et al., 2008). Several genetic and ecological studies revealed that several pathovars of $P$. syringae live on the plant surface, which frequently causes diseases and ice nucleation damage (Beattie and Lindow, 1999). $P$. syringae employs several strategies to cause disease, including quorum sensing, production of antimicrobial substances, suppression of plant survival defenses, 
bacterial multiplication, and occupation of the space normally occupied by other epiphytic species (Boch et al., 2002; Danhorn and Fuqua, 2007; Dulla and Lindow 2008; Monier and Lindow, 2005; von Bodman et al., 2003).

A starch-based super-absorbant polymer (SAP), referred to as Zeba ${ }^{\mathrm{TM}}$, has been used for soil amendment. In agriculture, SAP has been applied to help houseplants thrive with less water and fewer waterings. Due to the unique absorbing capacity of each particle, which absorbs up to 400 times its weight in water, SAP has also been used to improve seed germination, root development, and survival rate under conditions of water stress. In the present study, we elucidated that the water potential on the leaf surface plays a critical role in the early immigration and growth of epiphytic bacteria. In order to maintain free water, we applied a $0.1 \%$ starch-based SAP combination containing microbial nutrients to the leaves of Nicotiana benthamiana, a model host for studying plant pathogens (Krzymowska et al., 2008). In order to examine bacterial viability, we detected green fluorescent protein (GFP)-labeled microcolonies on leaves in situ under ultraviolet (uv) light using GFPuvtagged $P$. syringae pv. tabaci, which is a casual pathogen of wildfires on tobacco plants, including cultivars of $N$. tabacum and $N$. benthamiana. Intriguingly, macroscopic disease severity, when used as an indicator of bacterial virulence and population, was significantly different between leaves treated with the SAP combination and those treated with water (water control) following spray-inoculation, whereas bacterial cell numbers, elucidated following leaf tissue maceration and subsequent dilution plating, did not change significantly between the two treatments in repeated experiments. Collectively, the SAP combination promoted bacterial aggressiveness without changing bacterial cell numbers. To our knowledge, the present study is the first report showing the use of a SAP combination in a phyllosphere context.

\section{Materials and Methods}

Construction and evaluation of $P$. syringae pv. tabaciGFPuv. The pathogen used was $P$. syringae pv. tabaci (kindly provided by Joseph W. Kloepper, Auburn University, USA). The plasmid pDSK-GFPuv was obtained from Dr. Kiran Mysore at The S. R. Noble Foundation and transformed into $P$. syringae pv. tabaci by electroporation, and the fluorescent colonies were selected under longwavelength UV light using the standard protocol described previously (Wang et al., 2007). Prior to use, P. syringae pv. tabaci that harbored the pDSK-GFPuv plasmid was stored at $-80^{\circ} \mathrm{C}$ in King's $\mathrm{B}(\mathrm{KB})$ medium containing $20 \%$ glycerol. The strains were removed from ultra-cold storage, streaked onto $\mathrm{KB}$ plates that contained $25 \mu \mathrm{g} / \mathrm{ml}$ kanamycin, and incubated at $28^{\circ} \mathrm{C}$ for $24 \mathrm{~h}$ to check for purity. Single colonies were transferred onto $\mathrm{KB}$ plates that contained $25 \mu \mathrm{g} / \mathrm{ml}$ kanamycin and incubated for 2 days. Fully-grown bacteria were scraped off the plates and resuspended in sterilized distilled water (SDW). Based on the optical densities, the bacterial suspensions were adjusted to $10^{5} \mathrm{CFU} / \mathrm{ml}$. The starch-based SAP combination was composed (w/w or $w / v)$ of a milled powder of $50 \%$ $\mathrm{Zeba}^{\mathrm{TM}}$ (Chobi Ltd, Korea), $15 \% \mathrm{CaCl}_{2}, 5 \%$ sucrose, $10 \%$ softanol and white carbon as a $1: 1$ mixture, $5 \%$ potato dextrose broth, $5 \%$ nutrient broth, and 5\% tryptic soy broth. A $0.1 \%$ SAP combination was utilized for further experiment. Initially, SAP alone treatment was tested any side effect on plant leaves as well as total saprophytic bacterial number resulting in observing no difference between SAP alone and water treatments. Thus we excluded SAP alone treatment for further experiment.

Effect of the SAP combination on bacterial viability and aggressiveness. Tobacco seedlings ( $N$. benthamiana) were prepared as described previously (Ryu et al., 2007). Threeweek-old seedlings that germinated following direct seeding on soilless medium were transferred into plastic round pots (diameter $=10 \mathrm{~cm}$ ) that contained soilless medium. Two weeks after planting, the plants were challenged by spraying the leaves either with a mixture (1:1) of the SAP combination plus a suspension $\left(10^{5} \mathrm{CFU} /\right.$ $\mathrm{ml}$ ) of $P$. syringae pv. tabaci (pDSK-GFPuv) or with the pathogen alone as a pathogen control. $0,5,7$, and 14 days after the pathogen challenge, disease severity of the SAP+ $P$. syringae pv. tabaci (pDSK-GFPuv) and the sterilized distilled water $(\mathrm{SDW})+P$. syringae pv. tabaci (pDSKGFPuv) treatments were evaluated by measuring the bacterial number per leaf by plating the sap of macerated whole leaves on KB plates that contained $25 \mu \mathrm{g} / \mathrm{ml}$ kanamycin, which selected for the pDSK-GFPuv-containing bacteria, and then by counting the CFUs at $48 \mathrm{~h}$ after incubation at $27^{\circ} \mathrm{C}$, as well as counting the number of spots per leaf. Plants were grown at $25 \pm 3^{\circ} \mathrm{C}$ under natural light in a greenhouse. The experiments were repeated four times with at least 4 replications each time.

Data analyses. Data from each experiment were analyzed using JMP software (SAS institute, Cary, NC). The magnitude of the $\mathrm{F}$ value $(P \leq 0.05)$ was used to determine whether or not the treatments significantly affected disease severity or bacterial numbers. When a significant result of $\mathrm{F}$ test was obtained, Fisher's protected least significant difference post hoc tests were used to reveal significant differences between the means of the individual groups. 


\section{Results and Discussion}

Construction and evaluation of $P$. syringae pv. tabaciGFPuv. Typical wildfire symptoms were observed 5 to 7 days after spray-application of the $0.1 \%$ SAP combination and $10^{5} \mathrm{CFU} / \mathrm{ml}$ P. syngae pv. tabaci. Prior to symptom (bacterial spots) development, strong GFP-positive spots were detected under UV light (Fig. 1). The GFPuv system, as documented previously, can be utilized as a sensitive marker for monitoring bacterial population growth (Wang et al., 2007). Some GFP-positive spots were detected even before any apparent lesions, which indicated that this material can be applied as a nondestructive pathogenmonitoring system. However, the experiments with GFPuvtagged bacterium were restricted to a closed greenhouse because the release of genetically-modified bacteria into the

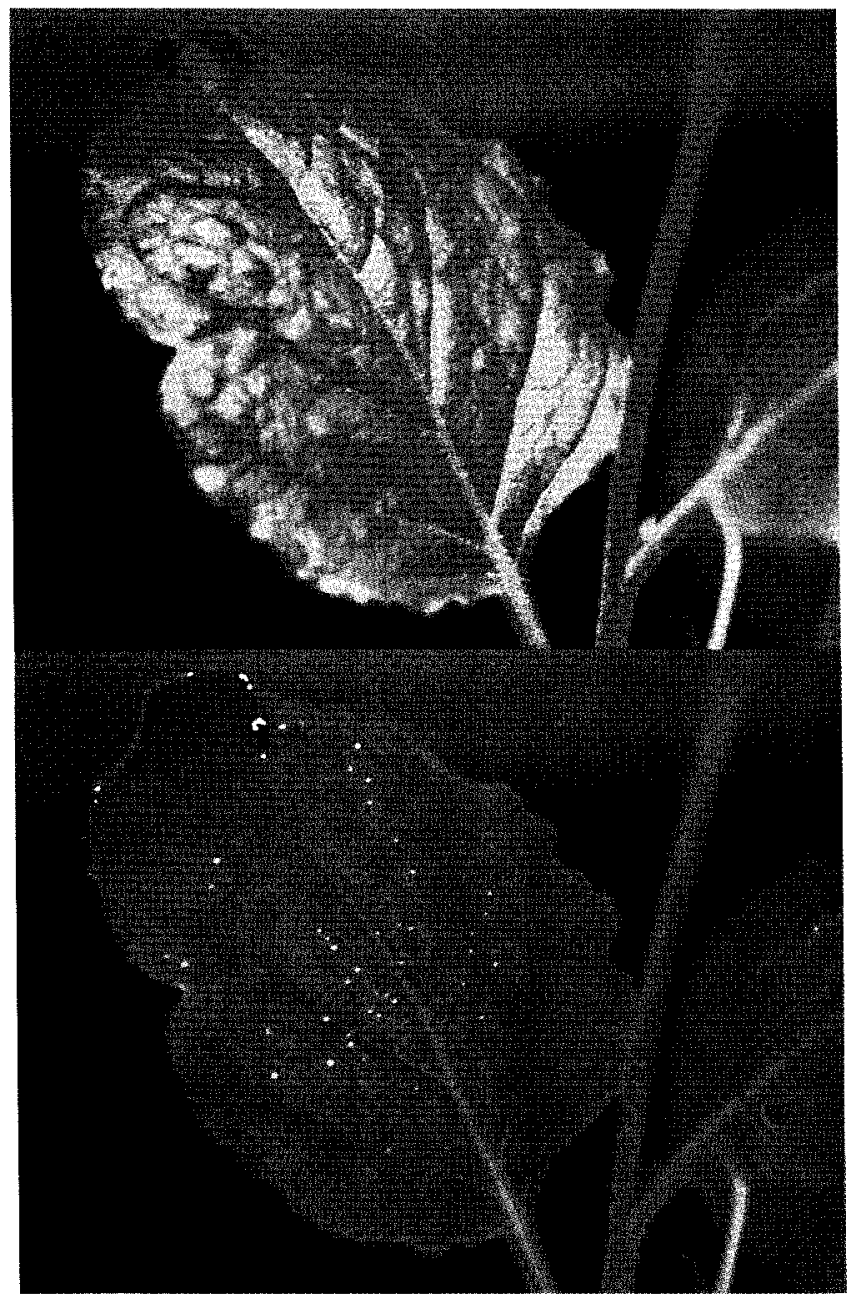

Fig. 1. Visualization of bacterial cells that colonized $P$. syringae pv. tabaci expressing GFPuv on the $N$. benthamiana leaf. (A) An $N$. benthamiana leaf shown under white light; slight chlorosis caused by $P$. syringae pv. tabaci was visible on the deep green leaf. (B) Under UV light, the GFP fluorescence of the $P$. syringae pv. tabaci microcolonies were located along the areas of chlorosis. nature is prohibited. Using this strain, Wang and associates (2007) previously showed a nondestructive method to detect bacterial populations by applying vacuum infiltration to $N$. benthamiana plants during virus-induced gene silencing of $N b S G T 1$. However, vacuum infiltration of $P$. syringae pv. tabaci (pDSK-GFPuv) into $N$. benthamiana leaves did not result in bacterial spot symptoms early in disease development, possibly because such a massive inoculation potential exists in nature. Recently, differences between spray and infiltration inoculation methods were reported (Melotto et al., 2007; Underwood et al., 2007). The authors showed that $P$. syringae colonized, entered leaves via the stomata on the plant leaf surface, and used the bacterial toxin coronatin to suppress abscisic acid (ABA) signaling that the plant normally used to control stomata closing. Consequently, these results suggest that the spraying of a GFP-tagged bacterial suspension for pathogen

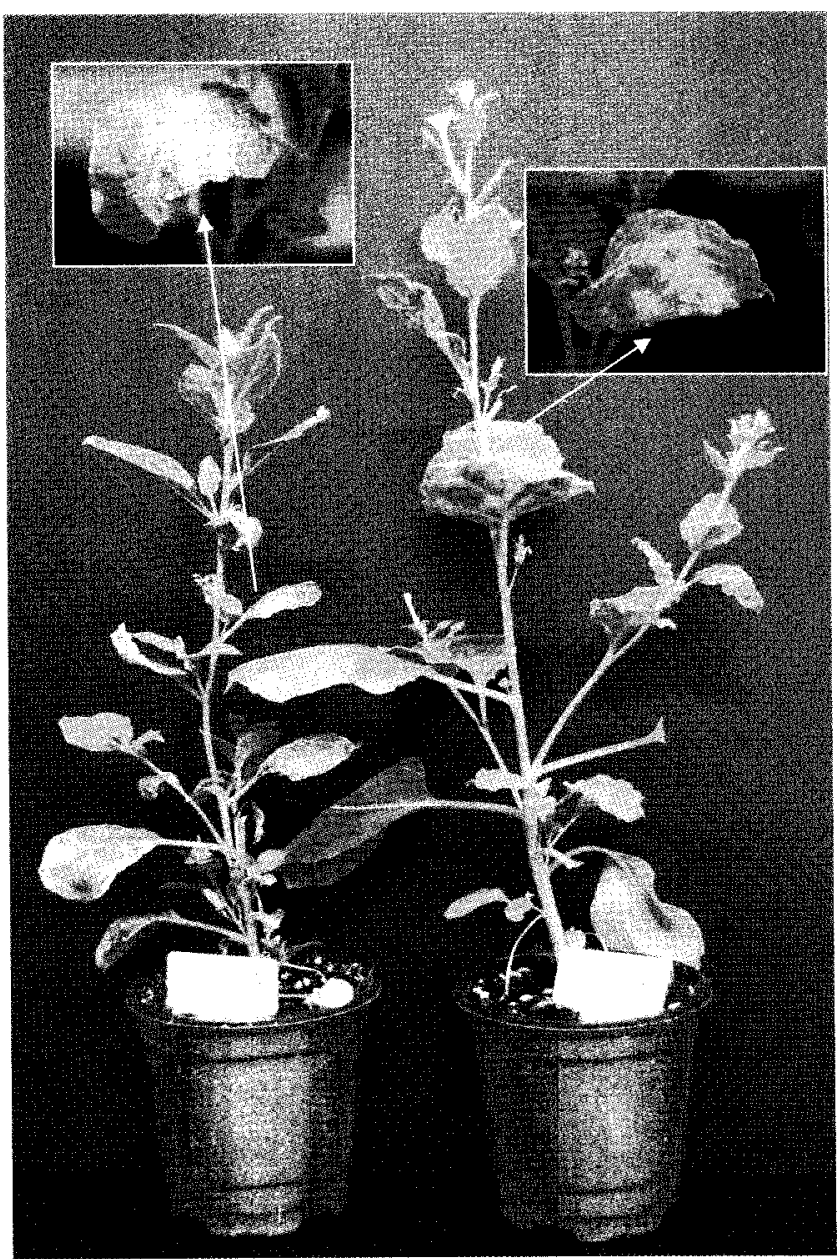

Fig. 2. Growth retardation due to the disease caused by $P$. syringae pv. tabaci in SAP-treated $N$. benthamiana at 14 days post inoculation. The growth of SAP-treated plants was reduced compared to water-treated plants. The severe disease symptoms of a representative SAP-treated plant are shown in detail in the small boxed region. 
inoculation might be a more appropriate method for studying pathogenesis of pathogenic bacteria in situ, whereas the infiltration method, as a pathogen challenge protocol, could prevent natural bacterial disease ecology in the phyllosphere.

\section{Effect of the SAP combination on symptom development.} At 14 days post inoculation (dpi), N. benthamiana treated with the SAP combination showed severe fire blight symptoms that progressed from bacterial spots to chlorosis and leaf death (Fig. 2). Normal plant growth was slightly inhibited in severely infected plants (Fig. 2). Plant height of the $N$. benthamiana plants treated with the SAP combination was consistently shorter than the height of watertreated plants (Fig. 2). Interestingly, we could not detect any significant differences in bacterial numbers between the leaves treated with the SAP combination and those treated by water at 5 and 14 dpi but do different between 5 dpi and $14 \mathrm{dpi}$ (Fig. 3). In the field, the growth of heavily infected tobacco plants is inhibited because chlorotic halos spread across the leaves, leading to leaf fall-off under conditions of high humidity, such as during the rainy season (Agrios 2000). The halo lesion is caused by production of the bacterial toxin, tabtoxin. We cannot exclude the possibility that the SAP combination either directly elicited tabtoxin secretion or optimized circumstances that favored production of the toxin. Mutation of the bacterial environmental receptor, GacS, in P. syringae pv. tabaci abolished pathogenesis and quorum sensing (Marutani et al., 2008). Quorum sensing by epiphytes plays a crucial role in leaf localization and retains the population (Danhorn and Fuqua, 2007).

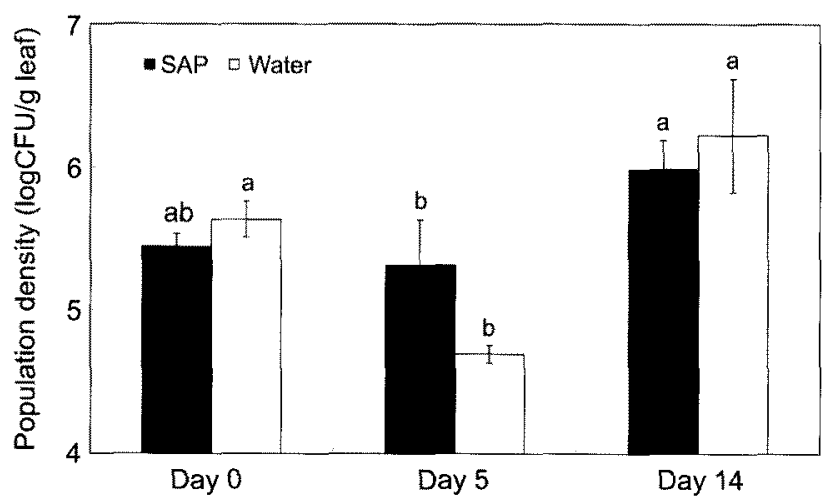

Fig. 3. Population density of $P$. syringae pv. tabaci in leaves of $N$. benthamiana at 0,5 , and 14 days post inoculation (dpi). Pathogen population density, both in SAP-treated and water-treated plants, was quantified on 0,5 , and 14 dpi. No statistical differences were observed between the SAP-treated plants and the water-treated plants. Different letters indicate significant differences between the treatments $(P=0.05)$. Error bars represent the standard errors of the mean values.
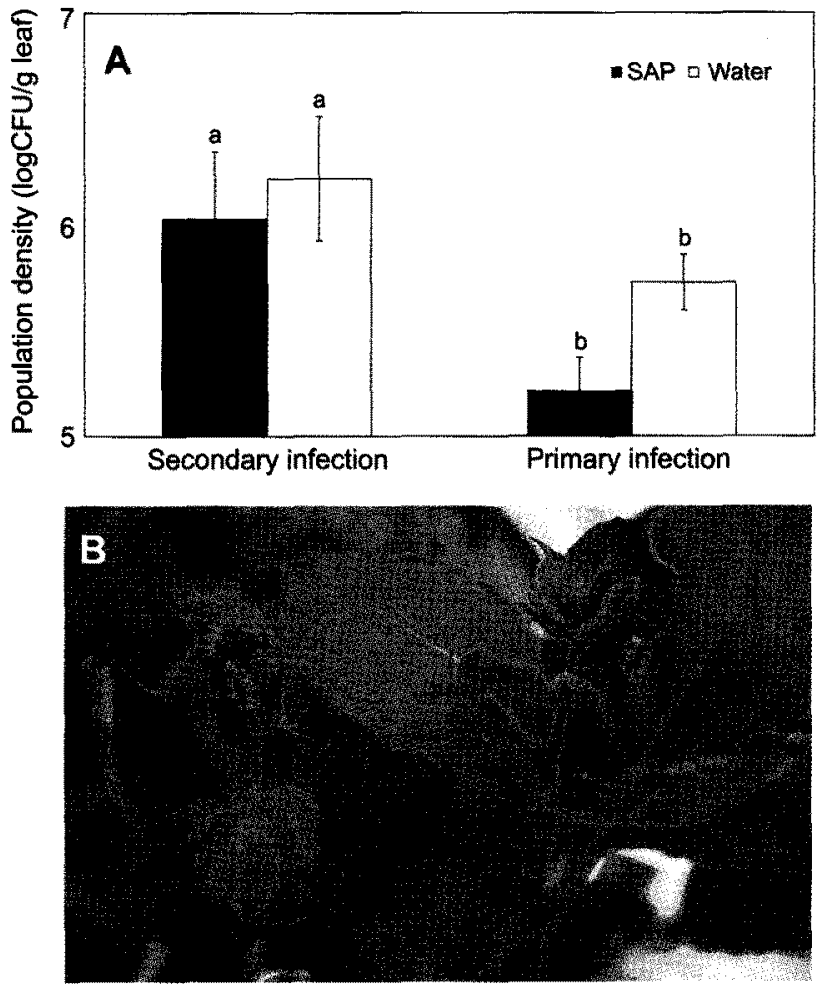

Fig. 4. Discrimination of disease symptom development and pathogen population density in the primary and secondary infection sites at 7 days post inoculation (dpi). (A) Pathogen population density in the secondary infection site of newly matured leaves and the primary infection site of existing leaves were quantified. Population density in the secondary infection site was higher than in the primary infection site, but there were no statistically significant differences between the SAP-treated plants and the water-treated plants. (B) Disease symptoms of the secondary infection site were worse in the SAP-treated plants than in the water-treated plants. (Left plant, SAP-treated plant; right plant, water-treated plant).

Detailed observations of bacterial aggressiveness. Preliminary experiments determined that the critical time point for examining the uncoupling of symptom severity from bacterial number on symptomatic leaves was 7 days after pathogen challenge (Fig. 4A, 4B). Moreover, at $7 \mathrm{dpi}$, symptoms of a secondary infection were apparent on the young leaves (Fig. 4B). There were no significant differences in bacterial numbers between the leaves treated with the SAP combination and those treated by water, but there was a significantly difference in bacterial numbers between leaves with primary or secondary infections (Fig. 4A) $(P=$ 0.05 ). The macroscopic disease symptoms significantly differed between leaves treated with the SAP combination and those treated by water. The differences in the symptoms were confirmed by counting the number of bacterial spots per leaf. The SAP combination that was applied together with the pathogen resulted in an above 6-fold increase in 


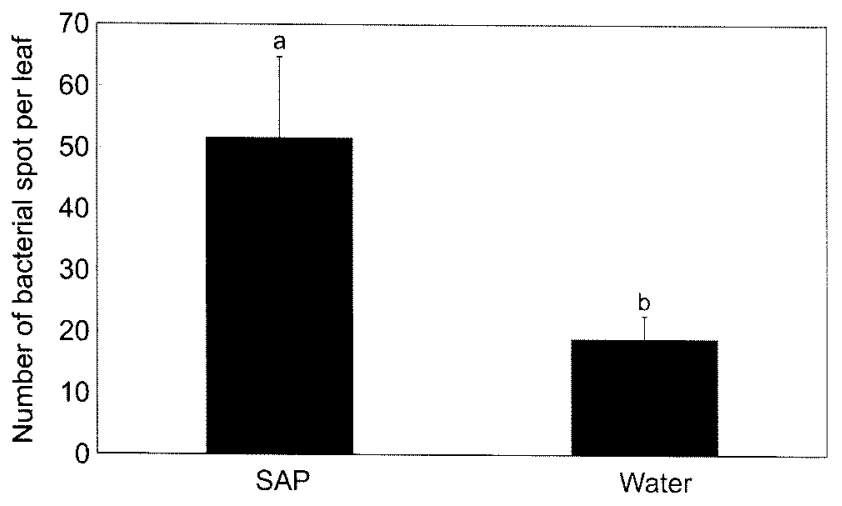

Fig. 5. Differences in the spot number of colonized bacteria that expressed GFPuv. Under UV light, bacterial spots that expressed GFPuv were counted. Different letters indicate significant differences between the treatments $(P=0.05)$.

spot number over the water control (Fig. 5). Previously, many researchers have used the dilution plating method to evaluate bacterial numbers following the infiltration of bacterial suspensions into the extracellular space of plant leaves and subsequent maceration of the plant tissues (Marutani et al., 2008; Wang et al., 2007). GFP-tagged plant pathogenic bacteria could be used as an additive and sensitive protocol to the maceration-dilution plating method as a way to nondestructively monitor bacterial population dynamics. Taken together, the SAP combination on the foliar parts of plants improved aggressiveness of a bacterial pathogen, $P$. syringae pv. tabaci. Furthermore, one advantage of the SAP combination would be its ability to enhance the efficiency of biological control agents against weeds or to confer a competitive advantage on saprophytic bacteria by helping them resist invasion by potentially harmful microbes.

\section{Conclusions}

During the evaluation of a SAP combination for the promotion of bacterial viability using GFP-tagged $P$. syringae pv. tabaci on the leaves of $N$. benthamiana, we obtained unexpected results. A clear distinction between symptom severity and bacterial cell number per leaf was consistently observed throughout repeated experiments. We hypothesize that the SAP combination somehow induced a bacterial virulence-related factor, such as the GacA/S two-component system. The results of this study indicate that the SAP combination application can promote the activities of biological control agents against weedy plants or increase the robustness of saprophytic epiphytes against potentially harmful microbes on leaves when applied in advance to development of disease symptom. In addition, we found that different methodologies, such as spray- vs. infiltration- inoculation, result in the detection of different numbers of bacteria on the plant leaves. The mechanisms behind these differences need to be examined in a future study.

\section{Acknowledgments}

We thank Kiran Mysore for providing a plasmid pDSKGFPuv and Joseph W. Kloepper for Pseudomonas syringae pv. tabaci. This work was supported by the Biogreen 21 (Code \#20070401034005) from the Korean Rural Development Administration, the Agricultural Research and Development Promotion Center (ARPC), and KRIBB initiative program in the Republic of Korea.

\section{References}

Agrios, G. N. 2005. Plant Pathology. $5^{\text {th }}$ ed. Academic Press. San Diego, CA, USA. 922 p.

Barak, J. D. and Liang, A. S. 2008. Role of soil, crop debris, and a plant pathogen in Salmonella enterica contamination of tomato plants. PLoS ONE 3:e1657.

Beattie, G. A. and Lindow, S. E. 1995. The secret life of foliar bacterial pathogens on leaves. Annu. Rev. Phytopathol. 33:145172.

Boch, J., Joardar, V., Gao, L. T., Robertson, L., Lim, M. and Kunkel, B. N. 2002. Identification of Pseudomonas syringae pv. tomato genes induced during infection of Arabidopsis thaliana. Mol. Microbiol. 44:73-88.

Danhorn, T. and Fuqua, C. 2007. Biofilm formation by plantassociated bacteria. Annu. Rev. Microbiol. 61:401-422.

Dulla, G. and Lindow, S. E. 2008. Quorum size of Pseudomonas syringae is small and dictated by water availability on the leaf surface. Proc. Natl. Acad. Sci. USA 105:3082-3087.

Goodin, M. M., Zaitlin, D., Naidu, R. A. and Lommel, S. A. 2008. Nicotiana benthamiana: Its history and future as a model for plant-pathogen interactions. Mol. Plant Microbe Interact. 21:1015-1026.

Gunasekera, T. S. and Paul, N. D. 2007. Ecological impact of solar ultraviolet-B (UV-B: $320-290 \mathrm{~nm}$ ) radiation on Corynebacterium aquaticum and Xanthomonas sp. colonization on tea phyllosphere in relation to blister blight disease incidence in the field. Lett. Appl. Microbiol. 44:513-519.

Heaton, J. C. and Jones, K. 2008. Microbial contamination of fruit and vegetables and the behaviour of enteropathogens in the phyllosphere: A review. J. Appl. Microbiol. 104:613-626.

Hirano, S. S. and Upper, C. D. 2000 . Bacteria in the leaf ecosystem with emphasis on Pseudomonas syringae-a pathogen, ice nucleus, and epiphyte. Microbiol. Mol. Biol. Rev. 64:624-653.

Kinkel, L. L. 1997. Microbial population dynamics on leaves. Annu. Rev. Phytopathol. 35:327-47.

Krzymowska, M., Konopka-Postupolska, D., Sobczak, M., Macioszek, V., Ellis, B. E. and Hennig, J. 2007. Infection of tobacco with different Pseudomonas syringae pathovars leads to distinct morphotypes of programmed cell death. Plant $J$. 50:253-264. 
Maduell, P., Armengol, G., Llagostera, M., Orduz, S. and Lindow, S. 2008. B. thuringiensis is a poor colonist of leaf surfaces. Microb. Ecol. 55:212-219.

Marutani M., Taguchi, F., Ogawa, Y., Hossain, M. M., Inagaki, Y., Toyoda, K., Shiraishi, T. and Ichinose, Y. 2008. Gac two-component system in Pseudomonas syringae pv. tabaci is required for virulence but not for hypersensitive reaction. Mol. Genet. Genomics. 279:313-322.

Melotto, M., Underwood, W., Koczan, J., Nomura, K. and He, S. 2007. Plant stomata function in innate immunity against bacterial invasion. Cell 126:969-980.

Monier, J. M. and Lindow, S. E. 2005. Spatial organization of dual-species bacterial aggregates on leaf surfaces. Appl. Environ. Microbiol. 71:5484-5493.

Ryu, C.-M., Kang, B. R., Han, S. H., Cho, S. M., Kloepper, J. W. Anderson, A. J. and Kim, Y. C. 2007. Tobacco cultivars vary in induction of systemic resistance against Cucumber mosaic virus and growth promotion by Pseudomonas chlororaphis O6 and its gacS mutant. Eur. J. Plant Pathol. 119:383-390.

Underwood, W., Melotto, M. and He, S. Y. 2007. Role of plant stomata in bacterial invasion. Cell Microbiol. 9:1621-1629.

von Bodman, S. B., Bauer, W. D. and Coplin, D. L. 2003. Quorum sensing in plant-pathogenic bacteria. Annu. Rev. Phytopathol. 41:455-482.

Wang, K., Kang, L., Anand, A., Lazarovits, G. and Mysore, K. S. 2007. Monitoring in planta bacterial infection at both cellular and whole-plant levels using the green fluorescent protein variant GFPuv. New Phytol. 174:212-223.

Whipps, J. M., Hand, P., Pink, D. A. and Bending, G. D. 2008. Human pathogens and the phyllosphere. Adv. Appl. Microbiol. 64:183-221. 